\title{
Synthetic adjacent pulse repetition interval length method to solve integer ambiguity problem: theoretical analysis
}

D. Wei

weidong@nanolab.t.u-tokyo.ac.jp

\section{K. Takamasu}

H. Matsumoto

\author{
Global Center of Excellence Program -Mechanical Systems Innovation-, School of Engineering, \\ The University of Tokyo, Hongo 7-3-1, Bunkyo-ku, Tokyo 113-8656, Japan \\ The University of Tokyo, Department of Precision Engineering, Hongo 7-3-1, Bunkyo-ku, Tokyo \\ 113-8656, Japan \\ The University of Tokyo, Department of Precision Engineering, Hongo 7-3-1, Bunkyo-ku, Tokyo \\ 113-8656, Japan
}

This paper describes a novel approach for realizing femtosecond optical frequency comb (FOFC)-based length measurement. This approach is based on the analogy between the phase unwrapping problem and the integer ambiguity problem. Because the conventional synthetic wavelength method can solve the former, we investigated the possibility of using a synthetic adjacent pulse repetition interval length method to solve the latter. The results of theoretical analyses and numerical investigations show the feasibility of the proposed method. Our results should contribute toward the further development of FOFC-based length measurement methods.

[DOI: http://dx.doi.org/10.2971/jeos.2013.13016]

Keywords: Instrumentation, measurement, optical frequency comb, interferometry, metrology

\section{INTRODUCTION}

Recently, numerous studies [1, 2] have focused on femtosecond optical frequency comb (FOFC)-based length measurement because this approach provides a highly accurate frequency reference for measurements. Length measurement using an FOFC source has distinct measurement characteristics [3, 4] and applications [5]-[7]. Many special techniques [8][11] have been developed to exploit these distinct characteristics (especially the temporal coherence) of FOFC light. A research group at the National Institute of Advanced Industrial Science and Technology has extensively studied length and length-related measurements using FOFC [12]-[16]. In particular, Minoshima and Matsumoto have pioneered the use of FOFC-based approaches for measuring large lengths with their original demonstration of the measurement of a $240-\mathrm{m}$ distance [12].

Figure 1 shows a schematic of the interference fringes obtained by a conventional Michelson interferometer with an FOFC light source. The correlation (autocorrelation and cross correlation) functions between two pulse trains afford two length scales: the central wavelength $\lambda_{c e n}$ and the adjacent pulse repetition interval length (APRIL) $c \times T_{R}$, where $c$ is the speed of light in vacuum and $T_{R}$, the pulse repetition period. When seeing one correlation function, the distance between the two peaks of the interference fringes can be understood to be half the central wavelength of FOFC. The size of one correlation function corresponds to the temporal coherence length of a single pulse, which is of the order of micrometers. In general, the central wavelength can be continuously used as the length scale within this range. When looking at the whole correlation functions, the distance between the two peaks of the envelope of the interference fringes can be understood to be half the APRIL of FOFC. In general, the size of the APRIL is of the order of meters. Furthermore, the existence of the APRIL is discrete. Below, we discuss how to perform length measurement by using the central wavelength and/or APRIL.

First, we consider the possibility of using the central wavelength of an FOFC as the length scale. Because the range of one correlation function is too short (of the order of micrometers) to cover a long distance (for example, of the order of meters), it is impossible to simply use the central wavelength as a length scale in an ordinary Michelson interferometer. We can extend the range to observe the correlation function by using multiple reference mirrors. However, because the target could be as much as around 10000 times larger than the range of one correlation function, the feasibility of this approach is low.

A single-wavelength helium-neon ( $\mathrm{He}-\mathrm{Ne})$ laser is used as a length standard. The use of an FOFC to produce a single wavelength appears promising because we can use a $\mathrm{He}-\mathrm{Ne}$ laser interferometer by simply changing the light source. However, a wavelength-based interferometer involves two main problems. The first one is the $2 \pi$ ambiguity. As in the case of a He-Ne laser interferometer, we can only detect the phase of the interference signal. The phase detection problem involves the $2 \pi$ ambiguity. The second one is the realization of a single wavelength.

Three schemes can be used to produce a single wavelength from an FOFC. The first scheme is to filter a single wavelength 


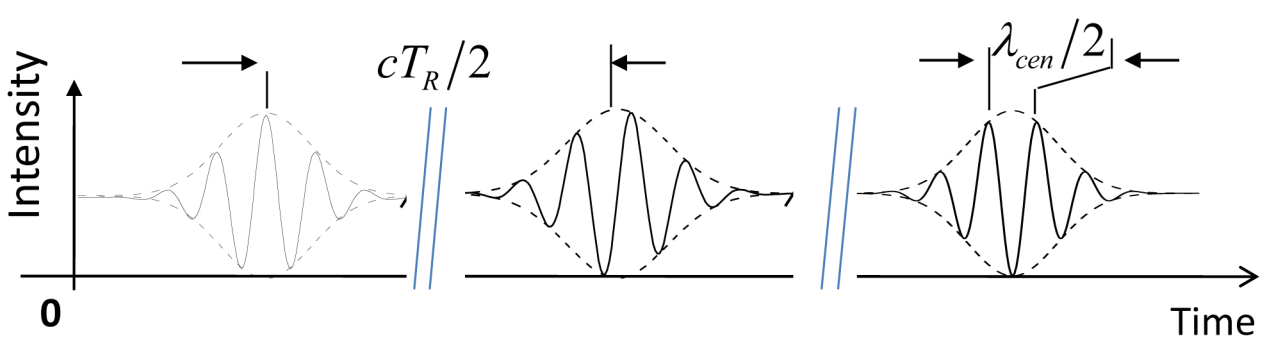

FIG. 1 Schematic of interference fringes formed by a multi-pulse train.

from an FOFC. The power of an FOFC is of the order of tens of milliwatts. Furthermore, an FOFC has numerous (of the order of millions) frequency elements (in other words, wavelengths). Therefore, the power of a single wavelength is of the order of nanowatts, which is too weak for practical measurements. The second scheme is to lock a single wavelength to the FOFC. For example, a laser diode was locked to an FOFC to achieve a single wavelength with strong power and high stability [17]. However, because we can only use a few frequencies from among the million or so that are available, the utilization efficiency of the FOFC is poor. The third scheme is to heterodyne two FOFC laser sources directly. By locking two FOFCs with different center frequencies, a single wavelength can be achieved as the bit signal (frequency difference) [18]. However, this scheme requires a complex feedback system. Therefore, further developments are required to achieve length measurement by using single wavelengths from an FOFC.

Next, we consider the possibility of using the APRIL of an FOFC as a length scale. The stability of an APRIL can be understood as follows. Because an APRIL is a set of superpositions of different single frequencies, if each frequency is stable, the APRIL is also steady at the same level. If the phase relationship among different frequencies is changed, it is impossible for the oscillation of the pulse train. The observation of the cross-correlation function shows that the phase relationship between frequencies is maintained. As long as the crosscorrelation function can be observed, an FOFC-based method can be used with APRIL as the length scale.

Studies have already demonstrated FOFC-based methods using an APRIL as the length scale by exploiting the stability of APRIL [5]-[7]. The autocorrelation function and the cross-correlation function of the pulse trains are detected simultaneously by combining two (an ordinary and an unbalanced) Michelson interferometers. The distance between the two peaks of the envelope of the autocorrelation function and the cross-correlation function can be expressed as a function of the APRIL. The signal is detected by intensity detection [5]-[7]. A heterodyne technique using an optical frequency comb to suppress the noise was presented in Ref. [5]. A FabryPerot etalon to change the APRIL of an optical frequency comb was reported in Ref. [6]. A heterodyne interferometer for position measurement with an acoustic-optical modulator and a piezo stage has been developed [7]. Generally, however, these methods suffers from the integer ambiguity (IA) problem. We have previously [3] used shutters set in front of the reflecting surfaces to turn the reflected light on and off in order to distinguish different reflecting surfaces. This paper presents a new approach to solve this IA problem for an absolute and arbitrary long-distance measurement.

The IA problem is studied from the viewpoint of the symmetry with the phase unwrapping (PU) problem. The remainder of this paper is organized as follows. Section 2 briefly reviews the PU problem and the synthetic wavelength method, and then, it describes the IA problem and the proposed method. Section 3 presents the numeral experiments. Finally, Section 4 summarizes the main conclusions and future studies.

\section{PRINCIPLES}

\subsection{Phase unwrapping problem and synthetic wavelength method}

The PU problem, which has been extensively studied previously [19]-[21], is only briefly reviewed here to introduce basic concepts. Assuming $h$ to be the distance between two points, the PU problem can be expressed as

$$
h=\lambda \varphi / 2 \pi=\lambda\left(\varphi_{N}+\varphi_{\Delta}\right) / 2 \pi
$$

Here, $\lambda$ is the wavelength of the light source used for the measurement, and $\varphi_{N} / 2 \pi$ and $\varphi_{\Delta} / 2 \pi$ are the integral part $\left(\varphi_{N} / 2 \pi\right.$ : integer $)$ and the excess fractional part $\left(0 \leq \varphi_{\Delta} / 2 \pi<1\right)$ of the phase $\varphi$, respectively.

By using the optical heterodyne measurement method [22], Fourier transform method [23], and fringe scanning method [24], we can measure the excess fraction part $\varphi_{\Delta}$. By using the optical heterodyne measurement method, because the beat frequency of two different frequencies is low, an excess fractional part can be detected by using a phase meter. The other two methods find an excess fractional part by using the $\tan ^{-1}$ [] function. The excess fractional part can be calculated assuming that $-2 \pi \leq \varphi_{\Delta}<2 \pi$ holds. A phase ambiguity corresponding to the integral part of the phase remains. The PU problem is nothing but the problem of finding the true value $\varphi$ from the excess fractional part $\varphi_{\Delta}$, i.e., finding $\varphi_{N}$.

Techniques for solving the PU problem using two or more wavelengths [25]-[30], called as the synthetic wavelength method, have already been introduced. We can understand this individual-point-based PU process as follows.

The distance $h$ can be expressed as follows by using two wave- 
lengths.

$$
h=\lambda_{i} \varphi_{i} / 2 \pi=\lambda_{1}\left(\varphi_{N 1}+\varphi_{\Delta 1}\right) / 2 \pi \lambda_{2}\left(\varphi_{N 2}+\varphi_{\Delta 2}\right) / 2 \pi
$$

Here, $\lambda_{1}$ and $\lambda_{2}$ are the two wavelengths. When $h$ is measured by using different wavelength $\lambda_{(i)}, \varphi_{N(i)}$ and $\varphi_{\Delta(i)}$ are the integral and the excess fractional parts of the phase $\varphi_{i}$, respectively.

By using two wavelengths, the following synthetic wavelength is obtained:

$$
\Lambda_{12}=\lambda_{1} \lambda_{2} /\left|\lambda_{1}-\lambda_{2}\right|
$$

Furthermore, $h$ can be expressed as

$$
h=\Lambda_{12}\left(\varphi_{N 12}+\varphi_{\Delta 12}\right) / 2 \pi
$$

For example, the synthetic frequency $\Lambda_{12}$ becomes $3.33 \mu \mathrm{m}$ when we assume $\lambda_{1}$ as $532 \mathrm{~nm}$ and $\lambda_{2}$ as $633 \mathrm{~nm}$. As described in previous studies $[19,20]$, when we know that $h<\Lambda_{12}$ in advance, we can estimate the integral part $\varphi_{N 12}$ based on the two excess fractional parts. Then, the true value of the distance $h$ can be unambiguously determined.

\subsection{Integer ambiguity problem and synthetic APRIL method}

When an arbitrary length $h$ is assumed to be a function of APRIL $A L$, it can be written as

$$
h=A L \times T=A L \times(N+\Delta)
$$

Here, $N$ and $\Delta$ are the integral and the excess fractional part, respectively.

In previous experiments, we showed that $\Delta$ can be measured from the distance between the peaks of the envelopes of the observed interference fringes in a multi-pulse train interferometer [31]. When measuring $h$ with a single APRIL, the IA problem occurs because we do not know the positional relationship between the two pulse trains that generate the crosscorrelation function.

From the symmetry between Eq. (1) and Eq. (5), we propose the synthetic APRIL (S-APRIL) method in which two different APRILs are used to produce a synthetic APRIL to solve the IA problem.

The distance $h$ measured by using two APRILs can be expressed as

$$
h=A L_{1} \times\left(N_{1}+\Delta_{1}\right)=A L_{2} \times\left(N_{2}+\Delta_{2}\right)
$$

Here, $A L_{1}$ and $A L_{2}\left(A L_{2}<A L_{1}\right)$ are the two APRILs. $N_{(i)}$ and $\Delta_{(i)}$ are the integral and the excess fractional part of APRIL $A L_{(i)}$, respectively.

The S-APRIL $A L_{12}$ is defined as

$$
A L_{12}=A L_{1} A L_{2} /\left|A L_{1}-A L_{2}\right|
$$

$A L_{12}$ becomes $12 \mathrm{~m}$ when $A L_{1}$ is $4 \mathrm{~m}$ and $A L_{2}$ is $3 \mathrm{~m}$.
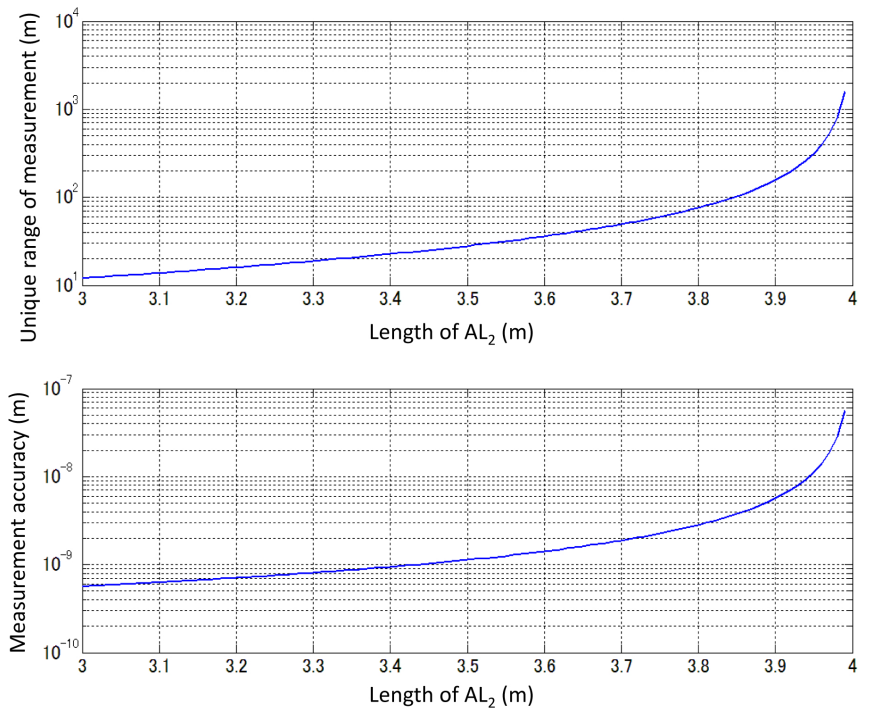

FIG. 2 Unambiguous range and achievable accuracy of S-APRIL method.

By using this S-APRIL, $h$ can be expressed as

$$
h=A L_{12}\left(N_{12}+\Delta_{12}\right)
$$

Inspired by [25]-[30], if we know that $h<A L_{12}$, as described below, the true value of an absolute length $h$ can be uniquely decided in the range of $A L_{12}$. We set

$$
\Delta_{12}=\Delta_{2} / A L_{2}-\Delta_{1} / A L_{1}
$$

Because $h<A L_{12}$, we have

$$
0 \leq\left(N_{12}+\Delta_{12}\right) / A L_{12}<1
$$

Because $0 \leq \Delta_{1} / A L_{1}<1$ and $0 \leq \Delta_{2} / A L_{2}<1$, we obtain $-1 \leq \Delta_{12}<1$. Therefore, we can obtain $h$ as

$$
h=\left\{\begin{aligned}
A L_{12}\left(\Delta_{12}\right), & 0 \leq \Delta_{12} / A L_{12}<1 \\
A L_{12}\left(1+\Delta_{12}\right) . & -1 \leq \Delta_{12} / A L_{12}<0
\end{aligned}\right.
$$

Next, we evaluate the accuracy of the S-APRIL method.

The accuracy of the S-APRIL obtained from Eq. (7) can be expressed as

$$
\delta A L_{12} / A L_{12}=A L_{12}\left(\delta A L_{12} / A L_{1}^{2}-\delta A L_{12} / A L_{2}^{2}\right)
$$

In general, we can assume that different APRILs are equally stable. We have $\delta A L_{1} / A L_{1}=\delta A L_{2} / A L_{2}=\delta A L / A L$, and Eq. (12) can be approximated as

$$
\delta A L_{12} / A L_{12} \approx\left[\sqrt{2} A L_{1} /\left(A L_{1}-A L_{2}\right)\right](\delta A L / A L)
$$

Nowadays, an FOFC with stability $\delta A L / A L<1 \times 10^{-10}$ can generally be easily achieved (for example, see [32]). When $A L_{1}$ is $4 \mathrm{~m}, A L_{2}$ is $3 \mathrm{~m}$ and $\delta A L_{12} / A L_{12} \approx 5 \times 10^{-10}$. In another case, when $A L_{1}$ is $4 \mathrm{~m}, A L_{2}$ is $3.99 \mathrm{~m}$ and $\delta A L_{12} / A L_{12} \approx 5 \times 10^{-8}$. Furthermore, the unambiguous range of the measurement becomes $1500 \mathrm{~m}$. Figure 2 show the unambiguous range (log scale) and the achievable accuracy (log scale) as a function of the length of the APRILs. In Figure 2, the horizontal axis indicates the length of $A L_{2}$ in meters $\left(A L_{1}\right.$ is $4 \mathrm{~m}$ and $\left.A L_{1}>A L_{2}\right)$. From Eq. (13), we understand that by improving the frequency stability, we can increase the measurement accuracy. 

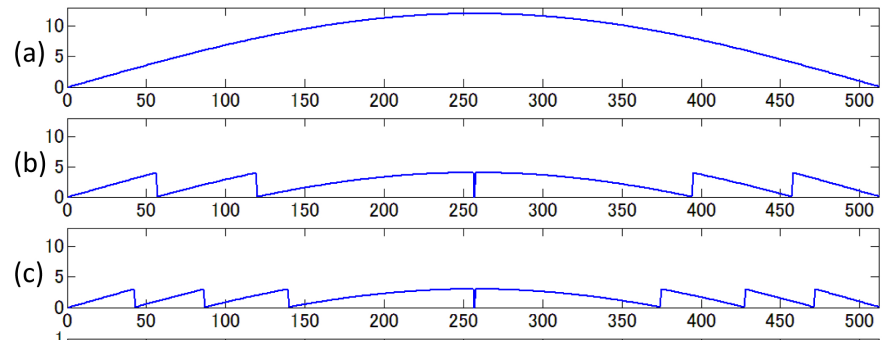

(d)
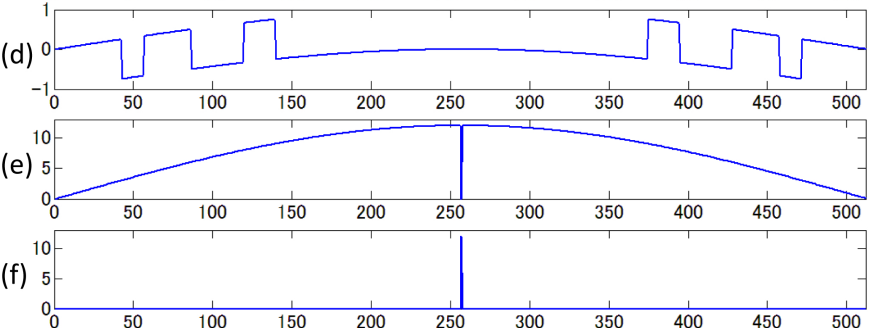

FIG. 3 Simulation of S-APRIL method. (a) is the object, $(b, c)$ is the excess fractional parts for different APRILs, (d) is the subtraction of the two excess fractional parts, (e) is the estimated length, (f) shows the difference between the object and the estimated length.

\section{NUMERAL SIMULATIONS}

Figure 3 shows the numeral simulation of the S-APRIL method. The object $x(n)$ measurement ( $n$ is the sample index) is considered to be a sin wave with amplitude of $12 \mathrm{~m}$, as shown in Figure 3(a). In Figure 3(a)-(c) and (e,f), the unit of the vertical axis is meters. The horizontal axis in Figure 3 indicates the sample index (arb. unit). The excess fractional parts $\Delta_{1}$ and $\Delta_{2}$ for APRILs for $A L_{1}=4 \mathrm{~m}$ and $A L_{2}=3 \mathrm{~m}$ are shown in Figure 3(b) and (c), respectively. The subtraction of the two excess fractional parts $\Delta_{12}$, as given in Eq. (9), is shown in Figure 3(d). In Figure 3(d), the unit of the vertical axis is arb. unit. Based on the value of $\Delta_{12}$, the measured length can be obtained from Eq. (11) as shown in Figure 3(e). To clarify the measurement accuracy, Figure 3(f) shows the difference between the object (Figure 3(a)) and the measured length (Figure 3(e)).

Figure 3(f) shows that we can accurately measure a length within $12 \mathrm{~m}$ and that if the value of the object is beyond the range of the synthesized APRIL, an error (peak in Figure 3(f) having an amplitude of $12 \mathrm{~m}$ ) occurs.

Because the measured excess fractional parts may be noisy, the length measurement for a practical real-world application is actually quite challenging. We discuss this noisy case by using the same computer simulation to illustrate the effects of noise on the estimation process of an integral part. Suppose that we have the discrete signal $x(n)$, and then, we add white noise $w n(x)$ to this signal as follows:

$$
x_{\text {noise }}(x)=x(n)+w n(n)
$$

Then, we measure the noisy signal as

$$
\Delta_{\text {noise }}(x)=I\left[x_{\text {noise }}(n)\right]
$$

Here, $I()$ is used to measure the excess fractional part. We assume that the two excess fractional parts share the same level but have different noise.

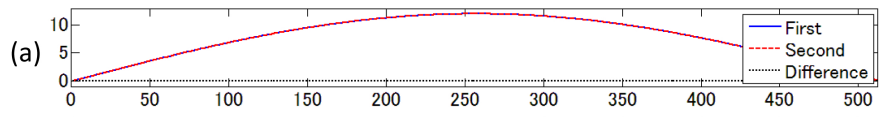

(b)

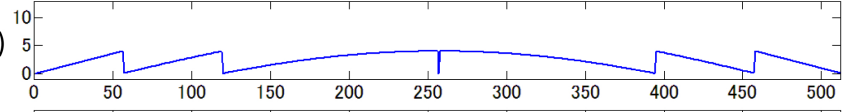

(c) 5

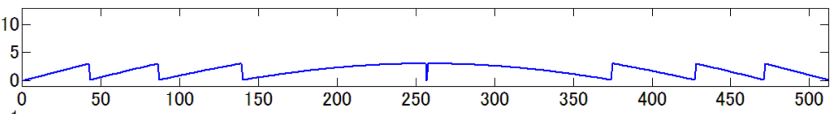

(d)
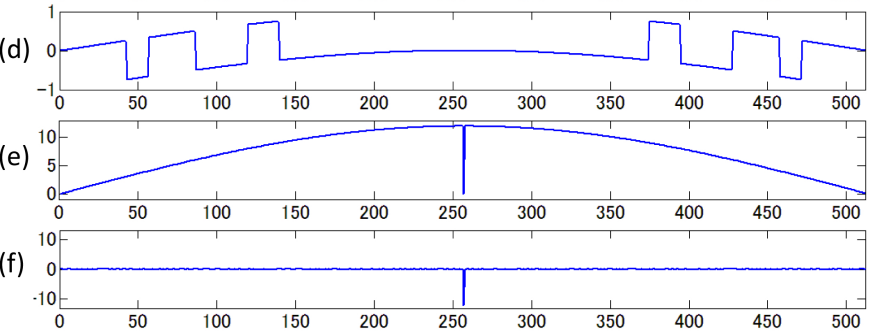

FIG. 4 Simulation for low level of noise. (a) is the two noisy object, $(b, c)$ is the excess fractional parts for different APRILs, (d) is the subtraction of the two excess fractional parts, (e) is the estimated length, ( $f$ ) shows the difference between the object and the estimated length.

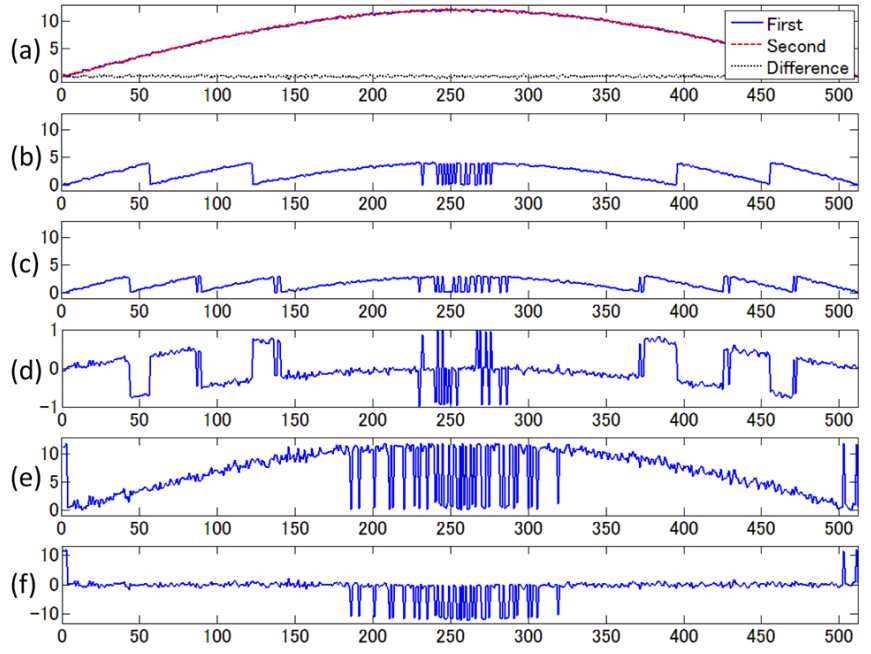

FIG. 5 Simulation for high level of noise. (a) is the two noisy object, $(b, c)$ is the excess fractional parts for different APRILs, (d) is the subtraction of the two excess fractional parts, (e) is the estimated length, ( $f$ ) shows the difference between the object and the estimated length.

We follow the same process to solve the noisy IA problem. First, we set the variance of the noise to a value of $10^{-6}$ which means that we can measure a distance of $1 \mathrm{~m}$ with an accuracy of the order of micrometers. The result is shown in Figure 4 . With the exception of Figure 4(a), each graph in Figure 4 shows the same calculation as that performed and shown in Figure 3. Figure 4(a) shows the two noisy signals (solid line and dashed line) and the difference between them (dotted line). The average value (without the value beyond the range of the S-APRIL) of the difference between the object and the measured length is $1.2 \times 10^{-6} \mathrm{~m}$, and its variance is $3.6 \times 10^{-9} \mathrm{~m}$. In this case, the noise does not adversely affect the estimation of the integral part.

The noise variance is now set to a higher value of $10^{-2}$ (Figure 5). We can observe that the higher noise level seriously affects the estimation of the integral part because many sig- 
nals (with true values near $0 \mathrm{~m}$ or $12 \mathrm{~m}$ ) exceed the unambiguous range of the measurement. Owing to the existence of a fake estimation, the estimation of the integral part of the signal, which is close to the measurement range, becomes quite challenging. The average value (without the value at which errors occur) of the difference between the object and the measured length is $0.008 \mathrm{~m}$, and its variance is $0.08 \mathrm{~m}$. We have found that in the case of a high level of noise, by selecting the unambiguous measurement range as being approximately two times of the target object, the S-APRIL method is feasible. For example, if the unambiguous measurement range is $12 \mathrm{~m}$, length measurement in the range from 3 to $9 \mathrm{~m}$ is very stable.

\section{CONCLUSIONS}

We introduced the symmetry of the PU problem and the IA problem. When an arbitrary length was measured as a function of the APRIL, from this symmetry, we proposed the synthetic APRIL (S-APRIL) method to determine the integer part. As a synthetic wavelength method that can eliminate the ambiguity of $2 \pi$ and determine an absolute and arbitrary short length (of the order of micrometers), S-APRIL can disambiguate the repeat interval and determine an absolute and arbitrary long length (of the order of meters). We confirmed the feasibility of the S-APRIL method and its measurement accuracy through a numeral simulation. The results of this study should contribute toward easy, highly accurate FOFC-based length measurements.

\section{ACKN OWLED GEMENTS}

This research work was financially supported by the Mizuho Foundation for the Promotion of Sciences and the Precise Measurement Technology Promotion Foundation, respectively. H. M. was supported by the "Development of System and Technology for Advanced Measurement and Analysis" Program at the Japan Science and Technology Agency.

\section{References}

[1] J. Ye, and S. T. Cundiff, Femtosecond optical frequency comb : principle, operation, and applications (Springer, New York, NY, 2005).

[2] W. Sibbett, A. A. Lagatsky, and C. T. A. Brown, "The development and application of femtosecond laser systems," Opt. Express 20, 6989-7001 (2012).

[3] D. Wei, S. Takahashi, K. Takamasu, and H. Matsumoto, "Analysis of the temporal coherence function of a femtosecond optical frequency comb," Opt. Express 17, 7011-7018 (2009).

[4] D. Wei, S. Takahashi, K. Takamasu, and H. Matsumoto, "Theoretical Analysis of Length Measurement Using Interference of Multiple Pulse Trains of a Femtosecond Optical Frequency Comb," Jpn. J. Appl. Phys. 50, 022701 (2011).

[5] H. Matsumoto, X. Wang, K. Takamasu, and T. Aoto, "Absolute Measurement of Baselines up to $403 \mathrm{~m}$ Using Heterodyne Temporal Coherence Interferometer with Optical Frequency Comb," Appl. Phys. Express 5, 046601 (2012).

[6] C. Narin, T. Satoru, T. Kiyoshi, and M. Hirokazu, "A new method for high-accuracy gauge block measurement using $2 \mathrm{CHz}$ repetition mode of a mode-locked fiber laser," Meas. Sci. Technol. 23, 054003 (2012).

[7] X. Wang, S. Takahashi, K. Takamasu, and H. Matsumoto, "Space position measurement using long-path heterodyne interferometer with optical frequency comb," Opt. Express 20, 2725-2732 (2012).

[8] J. Ye, "Absolute measurement of a long, arbitrary distance to less than anoptical fringe," Opt. Lett. 29, 1153-1155 (2004).

[9] M. Cui, M. G. Zeitouny, N. Bhattacharya, S. A. van den Berg, H. P. Urbach, and J. J. M. Braat, "High-accuracy long-distance measurements in air with a frequency comb laser," Opt. Lett. 34, 1982-1984 (2009).

[10] I. Coddington, W. C. Swann, L. Nenadovic, and N. R. Newbury, "Rapid and precise absolute distance measurements at long range," Nat. Photon. 3, 351-356 (2009).

[11] J. Lee, Y.-J. Kim, K. Lee, S. Lee, and S.-W. Kim, "Time-of-flight measurement with femtosecond light pulses," Nat. Photon. 4, 716-720 (2010).

[12] K. Minoshima, and H. Matsumoto, "High-Accuracy Measurement of 240-m Distance in an Optical Tunnel by use of a Compact Femtosecond Laser," Appl. Optics 39, 5512-5517 (2000).

[13] T. Yasui, K. Minoshima, and H. Matsumoto, "Three-Dimensional Shape Measurement of a Diffusing Surface by Use of a Femtosecond Amplifying Optical Kerr Gate," Appl. Optics 39, 65-71 (2000).

[14] Y. Yamaoka, L. Zeng, K. Minoshima, and H. Matsumoto, "Measurements and Numerical Analysis for Femtosecond Pulse Deformations After Propagation of Hundreds of Meters in Air with WaterVapor Absorption Lines," Appl. Optics 43, 5523-5530 (2004).

[15] Y. Bitou, T. R. Schibli, and K. Minoshima, "Accurate wide-range displacement measurement using tunable diode laser and optical frequency comb generator," Opt. Express 14, 644-654 (2006).

[16] M. Kajima, and H. Matsumoto, "Picometer positioning system based on a zoominginterferometer using a femtosecond optical comb," Opt. Express 16, 1497-1506 (2008).

[17] S. Hyun, Y.-J. Kim, Y. Kim, J. Jin, and S.-W. Kim, "Absolute length measurement with the frequency comb of a femtosecond laser," Meas. Sci. Technol. 20, 095302 (2009).

[18] S. Yokoyama, T. Yokoyama, Y. Hagihara, T. Araki, and T. Yasui, "A distance meter using a terahertz intermode beat in an optical frequency comb," Opt. Express 17, 17324-17337 (2009).

[19] C. R. Tilford, "Analytical procedure for determining lengths from fractional fringes," Appl. Optics 16, 1857-1860 (1977).

[20] G. L. Bourdet, and A. G. Orszag, "Absolute distance measurements by $\mathrm{CO}_{2}$ laser multiwavelength interferometry," Appl. Optics 18, 225-227 (1979).

[21] M. K. Kim, Digital holographic microscopy : principles, techniques, and applications (Springer, New York, 2011).

[22] R. Dändliker, B. Ineichen, and F. M. Mottier, "High resolution hologram interferometry by electronic phase measurement," 0 pt. Commun. 9, 412-416 (1973).

[23] M. Takeda, H. Ina, and S. Kobayashi, "Fourier-transform method of fringe-pattern analysis for computer-based topography and interferometry," J. Opt. Soc. Am. 72, 156-160 (1982).

[24] J. H. Bruning, D. R. Herriott, J. E. Gallagher, D. P. Rosenfeld, A. D. White, and D. J. Brangaccio, "Digital Wavefront Measuring Interferometer for Testing Optical Surfaces and Lenses," Appl. Optics 13, 2693-2703 (1974).

[25] H. Matsumoto, "Infrared He-Xe laser interferometry for measuring length," Appl. Optics 20, 231-234 (1981). 
[26] H. Matsumoto, "Synthetic interferometric distance-measuring system using a CO2 laser," Appl. Optics 25, 493-498 (1986).

[27] Y. Shuko, O. Jun, I. Shigeo, S. Katuo, M. Hirokazu, and S. Norihito, "Real-time and high-resolution absolute-distance measurement using a two-wavelength superheterodyne interferometer," Meas. Sci. Technol. 10, 1233 (1999).

[28] J. Gass, A. Dakoff, and M. K. Kim, "Phase imaging without 2? ambiguity by multiwavelength digital holography," Opt. Lett. 28 , 1141-1143 (2003).

[29] C. J. Mann, P. R. Bingham, V. C. Paquit, and K. W. Tobin, "Quantitative phase imaging by three-wavelength digital holography," 0 pt. Express 16, 9753-9764 (2008).
[30] A. Khmaladze, R. L. Matz, C. Zhang, T. Wang, M. M. Banaszak Holl, and Z. Chen, "Dual-wavelength linear regression phase unwrapping in three-dimensional microscopic images of cancer cells," Opt. Lett. 36, 912-914 (2011).

[31] D. Wei, S. Takahashi, K. Takamasu, and H. Matsumoto, "Time-offlight method using multiple pulse train interference as a time recorder," Opt. Express 19, 4881-4889 (2011).

[32] Y. Nakajima, H. Inaba, K. Hosaka, K. Minoshima, A. Onae, M. Yasuda, T. Kohno, et al., "A multi-branch, fiber-based frequency comb with millihertz-level relative linewidths using an intra-cavity electro-optic modulator," Opt. Express 18, 1667-1676 (2010). 